\title{
The Novel Technique for Fault Tolerance in WBAN
}

\author{
Harmandeep Kaur \\ YCOE, Punjabi University Campus Talwandi Sabo, \\ India-151302
}

\author{
Sukhwinder Singh Sran \\ YCOE, Punjabi University Campus Talwandi Sabo, \\ India-151302
}

\begin{abstract}
The wireless body area network is the other type of network which is used to sense the body conditions. In the wireless body area network, seven sensors are used with sense the whole human body conditions. These sensors sense the conditions and pass information to the transmitter. The main problem which occurred in the wireless body area network is FAULT. To recover fault ARP scheme is used. In this extra nodes deployed with the sensor nodes. The simulation results also verify the efficiency of proposed technique using three parameters energy consumption, routing overhead and throughput. The ARP improves 56\% energy as compared to existing method Routing protocol (RP). In addition, ARP approach improves throughput $18 \%$ delay.
\end{abstract}

\section{Keywords}

WSN, WBAN Protocols, WBAN network, Relay protocol.

\section{INTRODUCTION}

BAN is very powerful technique in wireless field which is uses in number of application for monitoring of health and fitness, device control and emergency response [2]. In this network there are some low profiles, low power devices that are interconnected in this way so that it creates sensing nodes consist of one and more sensor device known as microcontroller unit (MCU) and one radio transceiver that get rid of wire to make connection with coordinator nodes to transfer data from one node to other. Nowadays single chip of hardware is introduced in which transceiver and receiver in a single package according to the external components.

Basically sensors devices operate at access low level interface by loading MCU interfaces as a return it obtain data from actual sensor devices. It contains all the necessary instructions fetch from actual sensor devices. It also converts raw data into information which can be interpreted using logical information by using radio chip. These wireless nodes are worn and wrapped around human body [3].

A WBAN consist of convenient and independent sensor nodes for monitoring body movements for health, games, emergency, enjoyment applications.[13].Through the WBAN sensor nodes provide the patient better health monitoring services and provides carefully treatment. In body sensor nodes it allows connectivity between base station and receive station through remote monitoring. It is mainly make the most in Medical Application [4].

A WBAN consist of two parts one is in-body and other is onbody to monitor patient information regularly investigation and perception. Wireless Body Area Network traffic can be a categorized into On-demand, Emergency and Normal traffic [5].

On-demand traffic is mainly used doctors and co-ordinator to fetch data especially for diagnosis purpose. It can be continuous and discontinuous.

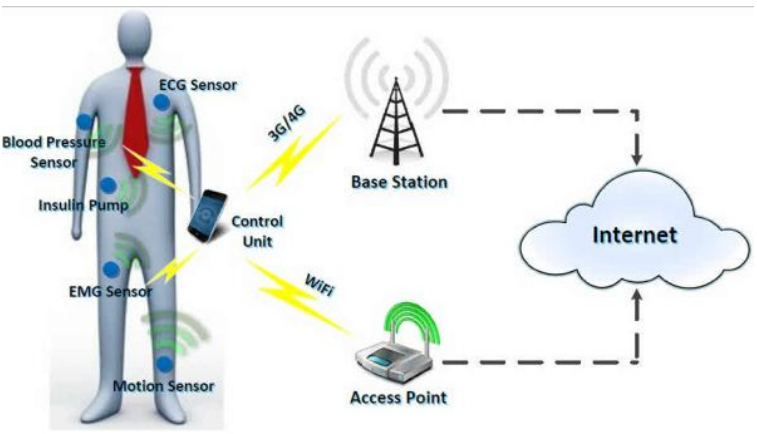

Fig 1: Body Area Network

Emergency traffic is initialized through the basic node when the threshold value is increased from the selected value in the seconds. Emergency traffic is not generated ordinary basis.

Normal traffic is provides the normal conditions of data transfer. In this traffic knows the patient all disease regularly basis and treatments. Data is collected through the receiver station.

\section{RELATED WORK}

Valenzuela et.al (2012) introduces [2] BAN in which for monitoring and identify internal activities of humans and animals through wireless sensor network and discuss about different categories of WBAN. Also provide the knowledge about design and deployment of wireless sensor network and issues about WBAN with external infrastructure. The technology, enable software and hardware and issues of WBAN is also discussed. Ullah et.al (2010) represents [1] that MEMS, combination of circuits and wireless communication have allowed to create the WBAN. WBAN provide best improvements in medical fields to detect the human body disease and provides real time updates and better improvement for patient disease. Wireless body area network provides military and healthcare applications. In this paper WBAN infrastructure shows the types of traffic and introduced how to protect these things. On demand, normal and emergency traffic are types of traffic which are used in infrastructure of WBAN. In this paper also focuses on antenna design for low power and MAC protocol for WBAN. These protocols are provide better energy efficiency to the body and on body sensor nodes. Limitation of Low power antenna design is also discussed. Pan et.al (2015) explains [3] that sensor node and body worn cordinator is harmful and poor to conditions of channel. This happens due to variations of sensing condition between sensor nodes and cordinators. The main solution of this problem is relay node where direct transmission is not possible. In this paper two hop relay meachiansm is proposed in IEEE 802.15.6 standard and divide it into relay node election, channel assesment, data relaying process. The main constraint of this process is it is initiated at different time interval which becomes reason of failure of data relaying. After this a predefined data relaying 
mechanism has been proposed to overcome data relaying failure problem. In this process predefined relaying node is activated without election. Experimental result shows that predefined relaying process improves $50 \%$ of packet delievery rate. Moreover network lifetime is also extended to $8 \%$. In future to improve packet delivery rate more, dynamic scheduling algorithm can be used. Arya and Bilandi (2014) reveal [4] the types of wireless body area network which are provide the detection and protection about health care system. These WBAN are mainly three types such as in body, on body and under body. In this paper also discuss MAC protocols and current technologies of WBAN. In this paper provide that how human beings and animals can get benefits through sensor nodes and provide better health care protection and identification. To improves health care system of India using Techniques and technology with the help of WSN without effecting of the patient. Khan et.al (2009) introduces [5] Wireless Body Area Networks which provide better way to protect the health care system. There are many applications of medical like health care, telecommunication, military, entertainment where WBAN can be used. In this paper we discussed the monitoring health care through attached the sensor nodes to the clothes of patients. Also discussed about the general system model for biosensor network and nodes are also attached inside the human body and also discuss the various issues and challenges of wireless body area netwok. Ragesh and Baskaran (2012) give [6] advancement in lower power integrated circuits and ultra lower power frequency, micro sensors allowed for the future of health care system using Wireless body area networks. In this system independent nodes are deployed on body parts, clothes and under skin. These technology is wide used for monitoring patient activities during illness. It can also be used in real time application like interactive gaming, consumer electronics, military etc. This technology never compell patients to sit in a hosiptal for physical mobility. This paper focus on various challenges of WBAN, secuirty issues, requirements, data security and important data context awareness. Heinzelman et al., (2002) [7] discussed about LEACH protocol. Along with the advancements in the battery and quality provided by nodes, network architecture had been a constant field of research. Recently, there has been much work on "poweraware" routing protocols for wireless networks. LEACH has been one of the basic routing protocols. In this protocol, data is sent along the optimum routes chosen. optimum routes are those which have more number of higher energy nodes. They may be longer in length. In LEACH, we use concept of dataaggregation and randomized rotation of the cluster head responsibility to avoid depletion of a single node of all its battery. Finally the author compares the results in terms of energy dissipation and latency with MTE (minimum transmission energy) routing. Hence LEACH is a protocol design where data is locally compressed and aggregated by cluster heads to trim down the size of shared data; network topology is set using local control, and media access control (MAC) and routing protocols; enabling a low-energy network overall. Mortaza et al., (2011) [8] proposed a new protocol which is actually the adapted version of LEACH with little enhancement. In this paper, the authors have tried their best to decrease the expenditure of the network resource in each round of choosing cluster head. Unlike LEACH, LEACH-C the cluster head choice takes place at base station. The location is divided into two phases. During the setup phase the base station receives location details and energy level of each node. It is responsibility of the base station to determine the node to be elected as cluster head based on the information about energy. Location information is utilised to form clusters by placing nearest nodes into one group. And the nodes whose energy is below than average node energy is removed from the list for becoming the cluster head.

\section{PROTOCOLS USED FOR WIRELESS BODY AREA NETWORKS}

There are various WBAN Routing protocols. These protocols are:

\subsection{Relay Protocols:}

In Relay protocols, high data rate nodes are placed and generate a structure of nodes. These nodes are arranged with manners and each node is communicated with among node. These nodes are transfer date one node to the another node through acknowledgement signal. Firstly setup all the nodes and create a circuit. Then transfer data from one node to another node, one node sends the request to another node then second node is send the acknowledgement signal to sender and data is transfer one node to another node. In the relay protocols sometimes nodes are dead by the degradation of battery and loss of their energy. Then data is not send source to destination. To overcome this disadvantage Advanced Relay Protocols are used in this research.

\subsection{Advanced Relay Protocols:}

In Advanced Relay Protocols firstly nodes are attached and create a circuit of nodes. In this circuit one node is sink node which is established in the center of another node. In this structure three extra nodes are established which are called relay nodes. These nodes are working for transfer the data when nodes are dead. When data is send from source to destination request and acknowledgement signals are transfer and then data is send to one node to another node. When one node is degrade and dead then nearby node is chosen for transfer the data. Relay nodes are chosen by great energy means that which node is highly energy and power occurred, that node is chosen for data transfer. So to recover the fault tolerance advanced relay protocols are used in this work.

\section{PROPOSED METHODOLOGY}

The wireless body area network is type of network which is used to sense the body conditions. The sensors are deployed in such a manner so that efficiently sensed data can be transmitted to transmitter. The communication in the wireless body area network is multi-hop communication. In this type of communication source node establish shortest path to destination. When source node is transmitting data to destination in between some sensor nodes get faulty or battery of the sensor nodes degraded due which fault may arise in the network. The relay based technique used relay nodes for fault recovery in the network. In this work, further improvement will be proposed in relay based network to reduce network complexity. The proposed enhancement will be based on dynamic routing. In this routing, multiple paths will be established from the source to destination. When battery of any sensor node reduced to threshold value, new path will be automatically chosen for data transmission. This technique may leads to reduce in network complexity, reduce network delay, reduce energy consumption and increase network throughput. The ARP improves 56\% energy as compared to existing method Routing protocol (RP). In addition, ARP approach improves throughput $18 \%$ delay.

There are number of nodes deployed in the network. Sink is also available in the network. Nodes transfer data through intermediate node to the sink. Node which wants to communicate with sink, data is transferred through 
intermediate node. During data transmission or forwarding data faults may be occurred at intermediate node in the network. Due to fault, network performance degrades in terms of throughput, packet loss, delay and energy. Transmission of data also affected due to fault occurrence.

\section{ALGORITHM}

\section{1: Start}

2: Initialize nodes

3: Setup Sender and Receiver nodes

4: for $\mathrm{i}$ in 0 to $\mathrm{n}$, where $\mathrm{n}$ is number of nodes

5: AODV $\leftarrow$ Node routing, Calculation of Routing

6: if (Route $S$ to $R$ found)

7: Check no. of route

8: end for

9: Find energy of each node

10: for $\mathrm{i}$ in 0 to $\mathrm{n}$

11: If(Energy $>20$ )

12: Select 3 only 3 nodes for best route and shortestpath

13: Send route acknowledgement

14: Nodes retvert back message to sender

15: Check higher energy node

16: Send data sender to receiver

\section{EXPERIMENTAL RESULTS}

To evaluate the results following parameters are used:

\subsection{Energy Consumption}

This refers to the total energy consumed in the network while transmitting the data packet. The energy left with the node is plotted against the simulation time in the network.

\section{Energy Consumption = Total Energy - Used Energy}

In this diagram shows the $\mathrm{X}$-axis is Time and $\mathrm{Y}$-axis is energy in joules. Red line is shown the proposed technique results and blue line shown the existing technique. This work is complete in 1 cycle and results are described. This figure shows the comparison of existing and proposed results. When simulation time is $2 \mathrm{sec}$. energy consumed by RP technique is 20 joule and ARP is 10 joule, at simulation time 4 sec. energy consumed by RP is 45 joule and ARP is 23 joule and so on.

Energy Consumption plot between Relay Protocol (RP) and Advanced Relay Protocol (ARP) scheme is shown below. In this case proposed technique saves $56 \%$ energy as compare to existing technique.

\section{Energy Consumption}

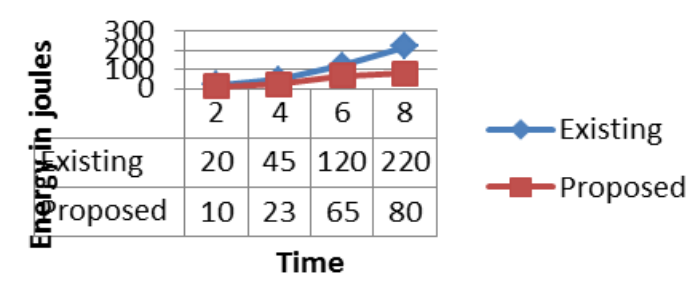

Fig 2: Energy Consumption
Table: 1 Comparison of Energy Consumption between RP (Relay Protocol) and ARP (Advanced Relay Protocol)

\begin{tabular}{|c|c|c|}
\hline $\begin{array}{c}\text { Simulation } \\
\text { Time }\end{array}$ & $\begin{array}{c}\text { Energy } \\
\text { Consumption(RP) }\end{array}$ & $\begin{array}{c}\text { Energy } \\
\text { Consumption(ARP) }\end{array}$ \\
\hline 2 & 20 & 10 \\
\hline 4 & 45 & 23 \\
\hline 6 & 120 & 65 \\
\hline 8 & 220 & 80 \\
\hline
\end{tabular}

\subsection{Throughput}

It is the rate of successful message delivery over a communication channel. Throughput is the maximum rate of production or the maximum rate at which something can be processed.

\section{Packet Size * Number of Packets Delivered Total duration of Simulation}

In this diagram shows the $\mathrm{X}$-axis is Time and $\mathrm{Y}$-axis is no. of packets. Red line and blue lines shows the existing technique and proposed work respectively. This work is complete in 1 cycle. This figure shows the comparison of existing and proposed results. When simulation time is $2 \mathrm{sec}$. throughput by RP technique is 8 and ARP is 12, at simulation time $4 \mathrm{sec}$. throughput by RP is 25 and ARP is 30 and so on. $18 \%$ throughput is increase comparison of existing technique.

Energy Consumption plot between Relay Protocol (RP) and Advanced Relay Protocol (ARP) scheme is shown below.

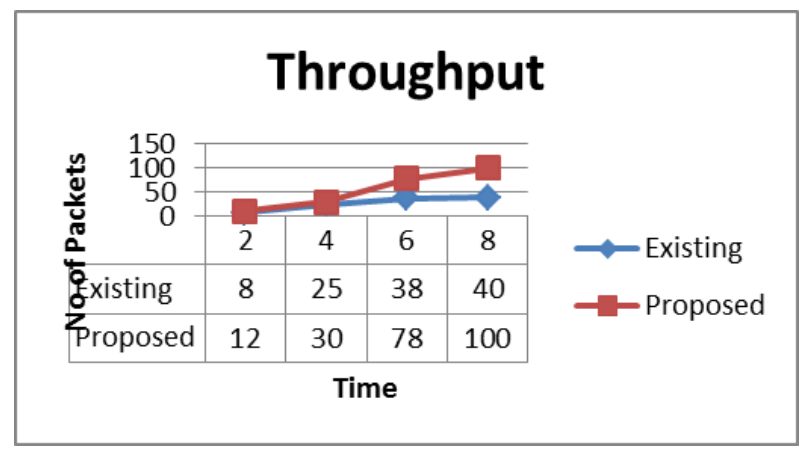

Fig 3: Throughput

Table: 2 Comparison of Throughput between RP (Relay Protocol) and ARP (Advanced Relay Protocol)

\begin{tabular}{|c|c|c|}
\hline $\begin{array}{c}\text { Simulation } \\
\text { Time }\end{array}$ & Throughput(RP) & Throughput(ARP) \\
\hline $\mathbf{2}$ & 8 & 12 \\
\hline $\mathbf{4}$ & 25 & 30 \\
\hline $\mathbf{6}$ & 38 & 78 \\
\hline $\mathbf{8}$ & 40 & 100 \\
\hline
\end{tabular}




\subsection{Routing Overhead}

Routing overhead refers to network routing information sent by an application, which uses a portion of the available bandwidth of a communication protocol or network.

In this diagram shows the two axis that are $\mathrm{X}$-axis is Time and $\mathrm{Y}$-axis is no. of packets. Red and blue lines are shows the proposed technique and existing technique results. This work is complete in 1 cycle and results are described. This figure shows the comparison of existing and proposed results. When simulation time is $2 \mathrm{sec}$. routing of RP technique is 30 means 30 packets are sent and ARP is 8 , at simulation time $4 \mathrm{sec}$. routing overhead by RP is 100 and ARP is 10 and so on.

Energy Consumption plot between Relay Protocol (RP) and Advanced Relay Protocol (ARP) scheme is shown below.

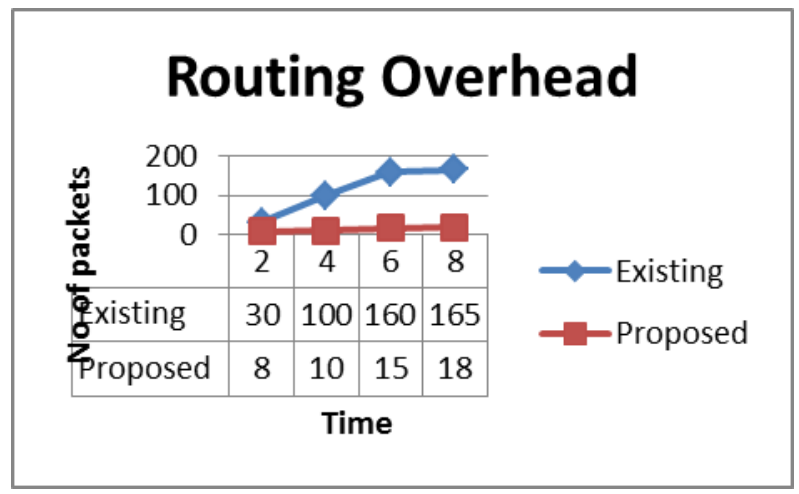

Fig 4: Routing

Table: 3 Comparison of Routing between RP (Relay Protocol) and ARP (Advanced Relay Protocol)

\begin{tabular}{|c|c|c|}
\hline $\begin{array}{c}\text { Simulation } \\
\text { Time }\end{array}$ & Routing(RP) & Routing(ARP) \\
\hline 2 & 30 & 8 \\
\hline 4 & 100 & 10 \\
\hline 6 & 160 & 15 \\
\hline 8 & 165 & 18 \\
\hline
\end{tabular}

\section{CONCLUSION}

The wireless sensor networks is the type of network which is used to sense the environmental conditions like temperature, pressure etc. The wireless body area network is used to sense the body conditions. In this work seven sensors are used with sense the whole human body conditions. These sensors sense the conditions and pass information to transmitter. In some cases fault is occur due to battery dead and threshold value. To recover fault ARP scheme is used. In this technique extra nodes deployed with the sensor nodes. These sensor nodes are chooses the greater node which is greater energy efficiency for reliable data transfer. Then data is transfer one node to another node. The simulation results also verify the efficiency of proposed technique using three parameters energy consumption, routing overhead and throughput. The ARP improves $56 \%$ energy as compared to existing method Routing protocol (RP). In addition, ARP approach improves throughput $18 \%$ delay. In future hybrid MAC protocol is used for greater energy and throughput and sends the greater data without any fault and disturbance.

\section{ACKNOWLEDGEMENTS}

The author would like to thank the Department of Computer Engineering, YCOE, Talwandi Sabo for providing platform for completing this research. I would also like thank all faculty members for giving inputs to compete this work.

\section{REFERENCES}

[1] Ullah, B., Saleem, and K. 2010. A Review of Wireless Body Area Networks for Medical Applications. International journal of Communication,Network and System Sciences(IJCNS).

[2] Gonza, L., Liang, H., and Chen, C. M. 2012. Body Area Networks",Autonomous Sensor Networks:Collective Sensing Strategies for Analytical Purposes. Springer Series on Chemical Sensors and Biosensors.

[3] Pan, D., Pathmasuntharam and Y. 2015. An Opportunistic Relay Protocol with Dynamic Scheduling in Wireless Body Area Sensor Network. IEEE Sensors Journal.

[4] Arya, N. 2014. A Review:Wireless Body Area Networks for Health Care. International Journal of Innovative Research in Computer and Communication Engineering.

[5] Ragesh, K. 2012. Addressing the Need for Context Awareness and Security Requirements in Wireless Body Area Networks. International Journal of Future Computer and Communication.

[6] Heinzelman, J. 1999. Adaptive Protocols for Information Dissemination in Wireless Sensor Networks. 5th Annual ACM/IEEE International Conference on Mobile Computing and Networking.

[7] Mortaza, M. A. 2011. Modify LEACH Algorithm for Wireless Sensor Network. International Journal of Computer Science Issues.

[8] Rupali, K. ,Ramesh, B., Deshmukh, B. 2013. Energy Management in Wireless Sensor Network. 15th International Conference on Computer Modelling and Simulation, IEEE.

[9] Qureshi, N., Khan, A., Akhtar, M. 2013 BEENISH:Balanced Energy Efficient Network Integrated Super Heterogeneous Protocol for Wireless Sensor Networks. ELSEVIER,Procedia Computer Science 19

[10] Lo, V. C. M. 2012. Wireless Body Area Network Node Localization Using Small-Scale Spatial Information. IEEE Journal of biomedical and Health Informatics. 